'Departamento de Ciencias y Tecnología Farmacéutica. Facultad de Ciencias Químicas y Farmacéuticas. Universidad de Chile. Santiago de Chile.

2Departamento de Medicina Intensiva, Facultad de Medicina. Hospital Clínico. Pontificia Universidad Católica de Chile. Santiago de Chile.

aQuímico Farmacéutico. Doctorando en Ciencias Farmacéuticas. Universidad de Chile. ${ }^{\text {b} Q u i ́ m i c o ~ F a r m a c e ́ u t i c o . ~ D o c t o r ~ e n ~ C i e n c i a s ~}$ Farmacéuticas. Universidad de Chile.

Este trabajo es parte del proyecto de investigación interdepartamental "Farmacocinética de antibimicrobianos en pacientes en shock séptico sometidos a hemofiltración de alto volumen, de los Departamentos de Medicina Intensiva y Nefrología de la Pontificia Universidad Católica, y parte del trabajo de Tesis Doctoral de uno de los autores (1a). Finaciamiento Dirección de Investigación Facultad de Medicina y Departamentos de Medicina Intensiva y Nefrología Facultad de Medicina Pontificia Universidad Catolica de Chile.

Recibido el 30 de junio de 2011, aceptado el 18 de octubre de 2011

Correspondencia a: Dr. Max Andresen Departamento de Medicina Intensiva. Facultad de Medicina. Hospital Clínico Pontificia Universidad Católica de Chile. Marcoleta $N^{\circ} 347$. Santiago de Chile. E-mail: andresen@med.puc.cl

\section{Consideraciones farmacocinéticas en el paciente crítico}

\author{
LESLIE ESCOBAR $^{1, \mathrm{a}}$, MARÍA NELLA GAI ${ }^{1, \mathrm{~b}}$, \\ TOMÁS REGUEIRA ${ }^{2}$ PhD, MAX ANDRESEN MSC ${ }^{2}$
}

\section{Pharmacokinetic considerations in critically ill patients}

Critically ill patients in Intensive Care Units (ICUs) are exposed to multiple procedures and usually require complex treatment regimens. Many of them suffer from comorbidities and different complications such as organ failure, drug-drug interactions, and unusual therapeutic interventions that can produce significant pathophysiologic changes. For that reason, pharmacokinetics for several substances is different to what is described for healthy patients, complicating drug selection and drug dosage to achieve appropriate effects. Low doses may determine a reduction of drug effectiveness and overdoses leading to toxicity. The aim of this paper is to review the pharmacokinetic considerations that must be considered when treating acute ICU patients.

(Rev Med Chile 2012; 140: 780-788).

Key words: Drug toxicity; Intensive care units; Pharmacokinetics.
E 1 término "farmacocinética" fue acuñado en los años treinta a raíz de estudios sobre la disposición de fármacos administrados por distintas vías. La relación existente entre la concentración plasmática de un fármaco en el tiempo, generada por los procesos ADME (absorción, distribución, metabolismo y excreción), ha conseguido actualmente utilizar la farmacocinética como una herramienta que permite aumentar la efectividad o reducir la toxicidad de una terapia, ya sea en un paciente individual o en un grupo especial de pacientes ${ }^{1}$.

En la Unidad de Cuidados Intensivos (UCI) la administración correcta de medicamentos es un desafío diario, dado que los cambios fisiopatológicos propios de los pacientes en estado crítico crean situaciones donde la información farmacocinética, obtenida de pacientes menos graves o sanos, no se ajusta a su situación.

Lo anterior se expresa como cambios en uno o varios parámetros farmacocinéticos, ya sea el volumen de distribución (Vd), el tiempo de vida media de eliminación $\left(t^{1 / 2}\right)$, la concentración máxima $\left(\mathrm{C}_{\max }\right)$ o el clearance $(\mathrm{Cl})$, entre otros. Esto, como consecuencia de disfunciones orgánicas, interacciones entre fármacos, respuesta de fase aguda o intervenciones terapéuticas agresivas, como por ejemplo, la reanimación con volumen o las terapias de reemplazo renal (TRR), tema de estudio de nuestro grupo de investigación. De ahí que el proceso $\mathrm{ADME}$ es particularmente diferente al descrito en situaciones normales (Tabla 1$)^{2-4}$. Por lo anterior, la aplicación de la farmacocinética clínica aparece como herramienta útil para optimizar la terapia farmacológica, limitando los efectos adversos por sobredosificación y/o evitando inefectividad por subdosificación en estos pacientes.

El objetivo de la presente revisión es describir y discutir aquellas situaciones propias del paciente en estado crítico que pueden alterar la respuesta terapéutica esperada debido a cambios en la farmacocinética. 
Tabla 1. Parámetros farmacocinéticos alterados en el paciente crítico

\begin{tabular}{|c|c|c|}
\hline \multicolumn{2}{|c|}{ Parámetro farmacocinético } & \multirow{2}{*}{$\begin{array}{l}\text { Situación en el paciente crítico } \\
\text { Alterada. Normalmente es menor porque la capa- } \\
\text { cidad de absorción disminuye }\end{array}$} \\
\hline $\begin{array}{l}\text { Biodisponibilidad } \\
\text { (BD) }\end{array}$ & $\begin{array}{l}\text { Porcentaje de la dosis que es capaz de alcanzar la } \\
\text { sangre luego de absorberse en el lugar de admi- } \\
\text { nistración del medicamento }\end{array}$ & \\
\hline $\begin{array}{l}\text { Unión a proteínas } \\
\text { plasmáticas }\end{array}$ & $\begin{array}{l}\text { Porcentaje de fármaco que está unido a proteínas, } \\
\text { como albúmina }\end{array}$ & $\begin{array}{l}\text { Disminuye. Frecuente reducción de la cantidad } \\
\text { de proteínas favorece un aumento la fracción de } \\
\text { fármaco libre }\end{array}$ \\
\hline $\begin{array}{l}\text { Volumen de } \\
\text { distribución }(V d)\end{array}$ & $\begin{array}{l}\text { Volumen donde se encuentra la cantidad de fár- } \\
\text { maco total administrado }\end{array}$ & $\begin{array}{l}\text { Aumentado. Mayor permeabilidad capilar y mayo } \\
\text { aporte de fluidos generan un tercer espacio de } \\
\text { distribución de fármacos }\end{array}$ \\
\hline Metabolismo & $\begin{array}{l}\text { Proceso del ADME que tiene por finalidad, hacer } \\
\text { excretables los fármacos administrados }\end{array}$ & $\begin{array}{l}\text { Disminuido. La respuesta será variable según los } \\
\text { metabolitos que se formen. Si son activos, es posi } \\
\text { ble que la respuesta disminuya. Si no, la respuesta } \\
\text { se prolongará en el tiempo }\end{array}$ \\
\hline $\begin{array}{l}\text { Vida media de } \\
\text { eliminación }(\mathrm{t} 1 / 2)\end{array}$ & $\begin{array}{l}\text { Tiempo en que la concentración plasmática de un } \\
\text { fármaco se reduce a la mitad }\end{array}$ & $\begin{array}{l}\text { Normalmente aumentada. Se relaciona inversa- } \\
\text { mente con el } \mathrm{Cl}\end{array}$ \\
\hline $\begin{array}{l}\text { Clearance renal } \\
\text { (Cl) }\end{array}$ & $\begin{array}{l}\text { Parámetro que da cuenta de la capacidad de elimi- } \\
\text { nación del fármaco desde la sangre. Volumen de } \\
\text { sangre que es depurada por minuto }\end{array}$ & $\begin{array}{l}\text { Variable. En las primeras etapas de trauma } \\
\text { sepsis, está aumentado. Posteriormente, podría } \\
\text { ocurrir falla renal que disminuya el } \mathrm{Cl}\end{array}$ \\
\hline
\end{tabular}

\section{Caracterización del ADME en el paciente crítico}

\section{Absorción}

La biodisponibilidad (BD) es el porcentaje de la dosis del fármaco administrado que alcanza la circulación sanguínea luego de absorberse. Sólo la administración endovenosa garantiza el 100\% de $\mathrm{BD}$, sin embargo, no todos los fármacos pueden administrarse por esa vía. Por esto, aquellos medicamentos que requieren absorción en el sitio de administración (vía enteral, intramuscular, subcutánea, sublingual, etc.) pueden afectarse particularmente en el estado crítico.

Cuando empleamos la vía enteral, la absorción del fármaco puede disminuir debido a cambios en la motilidad gastrointestinal o a reducción de la superficie de contacto de las mucosas. La primera situación puede afectarse por el uso concomitante de opiáceos, empleados frecuentemente para sedoanalgesia y por fármacos con efecto anticolinérgico, a modo de ejemplo, en intoxicaciones graves por antidepresivos tricíclicos. La segunda situación se debe a una disminución en la cantidad y altura de las microvellosidades intestinales, como consecuencia del ayuno prolongado y/o hipoperfusión, muy frecuente en los pacientes de $\mathrm{UCIs}^{2-4}$.
La redistribución del flujo sanguíneo hacia el cerebro, corazón y pulmones en estados de hipoperfusión, en desmedro de territorios como la piel, músculos, así como hígado o riñón, explica la menor BD encontrada tras la administración por vía dérmica, intramuscular o subcutánea ${ }^{2,4}$. Por ejemplo, el uso de enoxaparina subcutánea como prevención de trombosis en la dosis habitual de $40 \mathrm{mg}$, se asoció con niveles subterapéuticos de actividad anti-factor Xa causados por problemas de absorción en pacientes de $\mathrm{UCI}^{5}$.

\section{Distribución}

El volumen de distribución (Vd) es la relación entre la cantidad de fármaco total presente en un organismo y la concentración plasmática que esa cantidad genera. Es un parámetro propio de cada sustancia, según sus características fisicoquímicas (pKa, tamaño molecular, lipofilicidad/ hidrofilicidad, porcentaje de unión a proteínas plasmáticas, etc.) $)^{1}$. Algunas condiciones en el paciente crítico, como procesos inflamatorios sépticos o no sépticos, favorecen la salida de fluidos desde el espacio intravascular hacia el intersticial, por mayor permeabilidad capilar (capillary leak syndrome) creando un "tercer espacio"2,6,7 similar a lo que ocurre en casos de edema, ascitis o 
derrame pleural. Esta situación explica el mayor $\mathrm{Vd}$ para fármacos hidrofílicos como antibióticos $\beta$-lactámicos, glicopéptidos y aminoglicósidos, los que serán más abundantes en ese espacio que en el plasma $^{3,6,7}$. Ese desplazamiento del fármaco cambia el equilibrio de concentración plasma/tejidos, determinando que la concentración plasmática no sea el mejor indicador de las concentraciones alcanzadas. Con los antimicrobianos, lo más correcto sería determinarla en el sitio de la infección, sin embargo, son pocos los estudios disponibles con esa información. La propuesta de realizar microdiálisis en el tejido infectado y medir la concentración de antimicrobiano libre, aparece como una posible alternativa ${ }^{8}$. Otras situaciones que aumentan el Vd son la administración de fluidos (coloides o cristaloides) y la hipoalbuminemia. La primera aporta más volumen donde los fármacos se dispongan. En la segunda, una mayor fracción de fármaco libre podría penetrar a otros tejidos y distribuirse en ellos ${ }^{2,3,9}$.

\section{Metabolismo}

El principal objetivo del metabolismo de fármacos activos, liposolubles y no excretables, es convertirlos en hidrosolubles, inactivos y excretables ${ }^{1,10}$. El hígado es el principal órgano responsable del metabolismo y eliminación de muchos fármacos, identificándose dos fases en este proceso: Fase I, caracterizada por reacciones de oxidación que involucran al sistema enzimático citocromo P-450 (CYP) y la Fase II, conocida como fase de conjugación. Las enzimas de Fase I son menos abundantes que las de Fase II, y son afectadas en mayor medida por enfermedades o estrés celular, lo que las convierte en la etapa limitante del metabolismo de fármacos ${ }^{10}$. En el paciente crítico, la cantidad o actividad de estas enzimas se modifica por hipoxia, hipoperfusión hepática, liberación de citoquinas (presentes en procesos inflamatorios), estrés asociado a la hospitalización y también por los aportes nutricionales ${ }^{2,3,10}$.

Se describen muchos factores que alteran el metabolismo de fármacos, tanto ambientales como por interacción con otros fármacos (inhibidores o inductores enzimáticos), haciendo difícil predecir cómo será la biotransformación en el paciente crítico. Se ha propuesto estudiar la farmacocinética de los metabolitos de midazolam, ya que servirían como predictores del metabolismo hepático al requerir las enzimas de fase I y II para su formación ${ }^{3,10,11}$. En fármacos como remifentanilo y otros con metabolismo no hepático (extra-hepático), resulta más difícil saber cómo cambiará la biotransformación, independiente del paciente ${ }^{10}$.

\section{Excreción}

El clearance $(\mathrm{Cl})$, o aclaramiento, es el parámetro cinético que considera la sumatoria de todos los procesos que participan en la remoción y eliminación de la fracción libre del fármaco y sus metabolitos desde el plasma, siendo el componente renal el más importante para la mayoría de los fármacos $\left(\mathrm{Cl}_{\text {renal }}\right)$. Utilizando fórmulas como la Cockroft- Gault (CG) o Modification of Diet in Renal Disease (MDRD), se puede estimar la función renal según los niveles de creatinina plasmática y relacionar la capacidad de excreción de fármacos, siempre que la modificación de la creatininemia se deba principalmente a una disminución de la función renal ${ }^{12,13}$.

Conocemos que el deterioro agudo de la función renal se asocia a mayor morbimortalidad y es altamente frecuente en $\mathrm{UCI}^{14}$, aumentando con ello el riesgo de sobredosificación, llegando incluso a rangos tóxicos. De ahí que es necesario ser muy precisos en el ajuste de dosis según la función renal remanente ${ }^{12}$. No obstante, se ha observado un aumento del $\mathrm{Cl}_{\text {renal }}$ en ciertos pacientes críticos: grandes quemados, en estados hipermetabólicos o en las etapas iniciales del síndrome de respuesta inflamatoria sistémica, necesitándose mayores dosis para evitar concentraciones subterapéuticas, situación que normalmente es indetectable ${ }^{15}$.

En general, fármacos hidrofílicos o levemente lipofílicos y excretados por vía renal, deben ser considerados de alto riesgo de presentar fluctuaciones en sus concentraciones plasmáticas, requiriendo repetidos ajustes de dosis ${ }^{3,4,7}$.

En pacientes sometidos a terapias de reemplazo renal (como hemodiálisis, hemofiltración u otras), existe una importante excreción de fármacos ${ }^{16}$. Ésta se debe a factores dependientes del sistema de remoción (tipo de membranas, tamaño de poro y la capacidad de adsorción del filtro), de las características del fármaco (baja unión a proteínas plasmáticas, bajos $\mathrm{Vd}$ y $\mathrm{Cl}$ endógeno, alta hidrofilicidad y tamaño molecular pequeño) y de cambios farmacocinéticos del paciente (hipoalbuminemia, trastornos de permeabilidad capilar y alteraciones del Vd) las que generan concentraciones plasmá- 
ticas menores a las esperadas ${ }^{2,3,7,16}$. Muchos de los fármacos que comúnmente empleamos en terapia intensiva cumplen estos criterios, siendo los antibióticos los más estudiados, lo que sugiere fuertemente la necesidad de ajustes de dosis para lograr eficacia farmacológica durante todo el procedimiento $^{16}$.

Actualmente, la hemofiltración de alto volumen (HFAV) es un procedimiento de rescate hemodinámico cada vez más utilizado en $\mathrm{UCI}^{17}$. En nuestras unidades forma parte del manejo del shock séptico refractario ${ }^{14,18,19}$, pero aún falta precisar cómo esta técnica altera los procesos farmacocinéticos y, por ende, la dosificación de medicamentos. Por ejemplo, se ha visto que durante la HFAV en pacientes sépticos, el Cl de meropenem ocurre casi exclusivamente por la hemofiltración, siendo necesario administrar la dosis de $1 \mathrm{~g} / 8 \mathrm{~h}$ para mantener concentraciones adecuadas, a pesar del deterioro renal ${ }^{20}$. Otro ejemplo es vancomicina, glicopéptido de gran tamaño molecular, que es removida significativamente durante hemofiltraciones y hemodiafiltraciones ${ }^{21,22}$. Un estudio realizado en HFAV recomienda administrar una dosis equivalente a la de un paciente con función renal normal para asegurar una concentración efectiva, compensando el importante $\mathrm{Cl}$ extracorpóreo generado ${ }^{23}$.

Este comportamiento terapéutico hace pensar que existe el riesgo de concentraciones subterapéuticas de antibióticos, con una posible asociación a una falla terapéutica, pobres resultados clínicos y aumento en el desarrollo de resistencia bacteriana ${ }^{16}$.

\section{Índices farmacocinéticos y farmacodinámicos (PK/PD) para antimicrobianos}

Los antimicrobianos son el grupo terapéutico de mayor interés por varias razones: debido a que la sepsis grave y shock séptico son las principales causas de ingreso a UCIs, con una prevalencia de $33 \%$ en Chile $^{24}$ asociados a elevados costos económicos, utilización de muchos recursos sanitarios ${ }^{25}$ y alta morbimortalidad. Tampoco es posible medir directamente su efecto con algún parámetro clínico, como sí ocurre con otros fármacos (escalas de sedación, medición de presión arterial, glicemia, INR, etc.). Además sabemos que la administración oportuna y correcta determinaría una reducción de la mortalidad en la UCI y porque su uso inapropiado facilita la selección de cepas resistentes, acotando las alternativas terapéuticas actualmente disponibles ${ }^{26}$.

Para su efectividad, se requiere cumplir ciertas relaciones farmacocinéticas y farmacodinámicas (llamado PK/PD por Pharmacokinetics/Pharmacodinamics) entre la concentración plasmática alcanzada y el tiempo que esa concentración se mantiene sobre la concentración inhibitoria mínima (CIM) del patógeno causante de la infección ${ }^{6-8,16,27}$ (Tabla 2). La combinación de ambos parámetros puede permitir una aproximación sobre la respuesta terapéutica que se obtendrá, ya que ninguno por sí solo puede entregar información completa. La farmacocinética no entrega información de efectividad, y la farmacodinamia sobre la disposición de los antibióticos ${ }^{27}$.

Tabla 2. Relaciones farmacocinéticas/farmacodinámicas para los antimicrobianos

\begin{tabular}{|c|c|c|c|}
\hline Índice PK/PD & Clasificación & Descripción & Ejemplos \\
\hline $\mathrm{C}_{\max } / \mathrm{CIM}$ & Concentración-dependiente & $\begin{array}{l}\text { Relación entre la concentración máxima del } \\
\text { antibiótico y la CIM del patógeno causante } \\
\text { de la infección }\end{array}$ & Aminoglicósidos \\
\hline $\mathrm{T}>\mathrm{CIM}$ & Tiempo-dependiente & $\begin{array}{l}\text { Tiempo en que la concentración del anti- } \\
\text { biótico supera la CIM durante el intervalo } \\
\text { de administración }\end{array}$ & $\begin{array}{l}\beta \text {-lactámicos: Cefalosporinas } \\
\text { Carbapenémicos }\end{array}$ \\
\hline $\mathrm{ABC} / \mathrm{CIM}$ & $\begin{array}{l}\text { Concentración-dependiente } \\
\text { según el tiempo }\end{array}$ & $\begin{array}{l}\text { Relación entre el área bajo la curva de la } \\
\text { concentración del antibiótico durante } 24 \\
\text { horas, respecto a la CIM del patógeno cau- } \\
\text { sante de la infección }\end{array}$ & $\begin{array}{l}\text { Glicopéptidos: Vancomicina } \\
\text { Fluoroquinolonas }\end{array}$ \\
\hline
\end{tabular}

PK/PD: farmacocinética/farmacodinamia. $C_{\max }$ : Concentración máxima del antibiótico alcanzada durante el intervalo de administración. CIM: Concentración Inhibitoria Mínima. T: Tiempo. Intervalo de administración. ABC: Área bajo la curva en gráfico Concentración plasmática versus Tiempo. 
Según eso, los antibióticos se clasifican como concentración-dependiente o tiempo-dependiente (concentración independiente) dado por el gráfico de concentración plasmática/tiempo (Figura 1), que ha permitido hacer la terapia antimicrobiana más segura y efectiva ${ }^{4,27-29}$. Es la cantidad total administrada la que determina su eficacia ${ }^{27}$.

Los antibióticos concentración-dependiente (aminoglicósidos, fluoroquinolonas) deben alcanzar una concentración de fármaco libre al menos 10 veces mayor que la CIM del patógeno ${ }^{7}$ sin efecto tóxico para el paciente. Como presentan efecto post-antibiótico, luego de decaer la concentración plasmática siguen ejerciendo acción bactericida. Se describe una razón $\mathrm{C}_{\text {máx }}$ :CIM de 10:1 para aminoglicósidos. Asimismo, se requiere alcanzar una razón de área bajo la curva y la CIM (ABC:CIM) de 30:1 para quinolonas $s^{7,27,29}$.

En cambio, para los antibióticos tiempo-dependiente como los $\beta$-lactámicos, oxazolidinonas, macrólidos y glicopéptidos, la eficacia estará dada por el tiempo entre dosis en que las concentraciones de fármaco libre excedan la CIM, la que debe ser al menos durante el $50 \%$ del intervalo de dosificación, aunque en el paciente crítico o inmunocomprometido se espera sea en $100 \%$ del intervalo $^{3,27,29}$. De esta forma, se propone administrar altas dosis cada intervalos cortos y empleo de formulaciones de liberación sostenida o mediante infusiones continuas. Lo anterior permitiría disminuir las fluctuaciones de las concentraciones plasmáticas ${ }^{29,30}$, siempre que la estabilidad fisicoquímica del medicamento lo permitiera.

Aun cuando existan factores dependientes del paciente, sobre todo pacientes críticos, que modifiquen la disposición de los antibióticos, las propiedades fisicoquímicas de los antibióticos también son importantes para su utilización correcta (Tabla 3). Todo indica que en la práctica, se obstaculiza conseguir concentraciones plasmáticas esperadas.

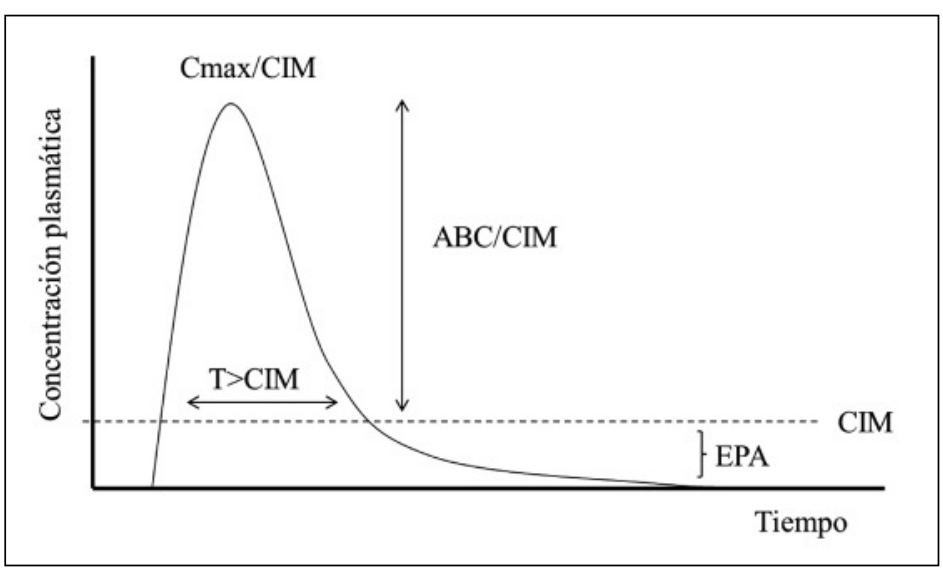

Figura 1. Parámetros farmacocinético/farmacocinámicos para antibióticos. CIM: Concentración inhibitoria mínima. EPA: Efecto post-antibiótico. T>CIM: Tiempo del intervalo de dosificación que la concentración del antibiótico supera la CIM. Cmax/CIM: Razón entre la concentración máxima y la concentración inhibitoria mínima. ABC/CIM: Razón entre el área bajo la curva y la concentración inhibitoria mínima.

Tabla 3. Clasificación de antimicrobianos según propiedades fisicoquímicas

\begin{tabular}{|ll|}
\hline Propiedad & Consideraciones \\
\hline Hidrofílicos & Vd normalmente bajo, pero susceptibles de modificarse por los factores que \\
$\beta$-lactámicos: Penicilinas, cefalosporinas, & afectan el $\mathrm{Vd}$ en el paciente crítico. Difícilmente atraviesan membranas, por lo \\
carbapenémicos, monobactamos & $\begin{array}{l}\text { que no tienen penetración intracelular. Eliminación principalmente renal, casi } \\
\text { inalterados en orina }\end{array}$ \\
$\begin{array}{l}\text { Amicopéptidos } \\
\text { Lipofílicos }\end{array}$ & \\
$\begin{array}{l}\text { Macrólidos } \\
\text { Fluoroquinolonas } \\
\text { Tetraciclinas }\end{array}$ & Gran Vd, fuera del espacio vascular. Buena penetración intracelular. Requieren \\
Vd: Volumen de distribución. & metabolismo normalmente hepático para ser excretados \\
\hline
\end{tabular}


Suelen emplearse asociaciones de $\beta$-lactámicos junto a glicopéptidos o aminoglicósidos durante los primeros días de tratamiento hasta identificar el patógeno, y luego desescalar al más específico. Sin embargo, con dosis adecuadas según la función renal del paciente, se ha demostrado que se obtienen concentraciones plasmáticas insuficientes con las primeras administraciones de $\beta$-lactámicos, sin lograr superar la CIM de Pseudomona aeruginosa $a^{30}$. Esta información ha motivado proponer estudios farmacocinéticos al ingreso de los pacientes a UCI, para dosificar los antibióticos pese a todas las variables fisiopatológicas que conducen a concentraciones inefectivas ${ }^{31}$.

Un estudio de monitorización de imipinem en una UCI pediátrica reveló una baja dosificación en $30 \%$ de los casos junto a una gran variación inter-individual de los parámetros farmacocinéticos $^{32}$, situación también observada en adultos ${ }^{33}$, concluyéndose que el monitoreo de fármacos debería ser frecuente y no basarse exclusivamente en algoritmos para el ajustar las dosis en pacientes inestables ${ }^{4,6-8,27-29}$.

Otro ejemplo es cefepime, que en dosis de $2 \mathrm{~g} / 12 \mathrm{~h}$ es apropiado y seguro en pacientes con $\mathrm{Cl}_{\text {crea }}>50 \mathrm{ml} / \mathrm{min}$. Sin embargo, esta dosificación es baja en $50 \%$ de los pacientes infectados con gérmenes con CIM $>8 \mathrm{mg} / \mathrm{L}$ con igual riesgo de toxicidad neurológica ${ }^{34}$.

Actualmente, la simulación de Montecarlo ha integrado parámetros PK, PD y de CIM del patógeno como una aproximación más real para definir las dosis ${ }^{28}$.

\section{Monitorización de antibióticos}

Por lo expuesto, para optimizar una terapia con antibióticos no sólo se deben considerar los parámetros $\mathrm{PK} / \mathrm{PD}$, también es necesaria la monitorización debido a la alta variabilidad interindividual en los pacientes. Esto ha llevado al desarrollo simulaciones y modelos farmacocinéticos en subgrupos específicos de pacientes, sin embargo, la experiencia es aún limitada ${ }^{28}$.

De esta forma, cuando apliquemos la farmacocinética en la práctica clínica podremos realizar una dosificación acorde a las necesidades de nuestros pacientes, pareciendo ser costo-efectiva para ciertos grupos terapéuticos como lo son los aminoglicósidos $^{35}$. Se ha sugerido monitorizar aquellos fármacos con estrecho margen terapéutico, que se asocian a un alto riesgo de toxicidad o con alta variabilidad de las características farmacocinéticas. Así, pacientes con función renal deteriorada, con variaciones del Vd como grandes quemados, sépticos o pacientes obesos, debieran ser candidatos para medir niveles de aquellos fármacos no evaluables por respuesta clínica, con el objetivo de individualizar la dosis a partir de la interpretación de los valores de su concentración plasmática ${ }^{1,36,37}$. En el caso de los antibióticos, la monitorización puede ser la herramienta que caracterice el mejor esquema de dosificación para lograr eficacia y evitar aparición de resistencia, independiente si el antibiótico en cuestión es de estrecho margen terapéutico o no.

No obstante, la interpretación de las concentraciones obtenidas es discutible. La variabilidad en los procesos de obtención de las muestras (tiempos, intervalos, dosificación, etc.) y las metodologías analíticas disponibles son factores limitantes para generar datos adecuados, lo que dependerá de cada laboratorio clínico ${ }^{36,38}$ y del conocimiento en farmacocinética de quien reciba esa información.

La monitorización terapéutica se realiza rutinariamente para dosificar aminoglucósidos y glicopéptidos entre otros, pero podría ofrecer ventajas terapéuticas para más fármacos, siempre que se cuente con las metodologías analíticas apropiadas $^{36}$.

Para vancomicina, se recomienda medir concentraciones séricas una vez a la semana para pacientes hemodinámicamente estables y con más frecuencia para aquellos más graves, justo antes de la siguiente dosis (nivel valle), luego de haber alcanzado el steady-state o estado de equilibrio (después de la cuarta dosis), siempre y cuando la administración se realice 2 ó 3 veces/día ${ }^{22,36,37,39}$. En pacientes con $\mathrm{VFG}<10 \mathrm{~mL} / \mathrm{min}$, debiera realizarse a las $48 \mathrm{hrs}$. Se espera alcanzar una concentración $\geq 10 \mathrm{mg} / \mathrm{L}$ para evitar el desarrollo de resistencia y una relación $\mathrm{ABC} / \mathrm{CIM}>400^{22,38,39}$. Con una concentración valle de 15 a $20 \mathrm{mg} / \mathrm{L}$ mejoraría la penetración en tejidos y con ello, la respuesta clínica.

\section{Otras aplicaciones de la farmacocinética} clínica

La Target Concentration Intervention (TCI) a diferencia del Therapeutic Drug Monitoring (TDM), consiste en modificar la dosis de un medi- 
camento a tiempo real para optimizar la respuesta de un paciente individual, alcanzando los niveles de concentración esperados según datos farmacocinéticos poblacionales ${ }^{40,41}$. En ese escenario, se ha propuesto cambiar la forma de administrar algunos medicamentos en UCI, pasando de bolo intravenoso a infusión continua programada, principalmente para opioides, propofol y otros anestésicos de acuerdo a un modelo farmacocinético definido que genere concentraciones estables en el tiempo o que puedan ser modificadas rápida y fácilmente ${ }^{40}$, ya que conseguir un equilibrio entre sedación excesiva o insuficiente es un desafío constante en la $\mathrm{UCI}^{42}$.

El primer ejemplo de esta aplicación fue la administración de propofol. Dependiendo de la edad, sexo y el peso del paciente, se administran continuamente dosis que generen concentraciones plasmáticas estables, según un modelo farmacocinético tricompartimental ${ }^{40}$.

También midazolam se utiliza frecuentemente dado su efecto sedante, amnésico y rápido inicio de acción, pero se describe una alta variabilidad inter-individual en su respuesta, tanto por acumulación en falla renal o por interacción con otros fármacos que inhiben su metabolismo hepático, teniendo variable vida media de eliminación ${ }^{11,41-43}$.

Así, se ha propuesto administrar un bolo de carga seguido de una infusión continua para lograr concentraciones plasmáticas más estables, con un efecto controlado ${ }^{44}$.

Para vancomicina, la infusión prolongada no ha mostrado tener mejor efectividad comparado con las infusiones intermitentes ${ }^{39}$, caso contrario para $\beta$-lactámicos, con los que sí se han visto ventajas $^{45}$.

\section{Conclusiones}

Se ha observado que las alteraciones fisiopatológicas que manifiestan los pacientes críticos, pueden generar cambios significativos y dinámicos en uno o en varios de los cuatro procesos farmacocinéticos (ADME). Lo anterior se ha expresado como gran variabilidad entre pacientes frente a iguales dosis administradas.

Debido a esto, es necesario individualizar las terapias de medicamentos que pueden salvar vidas, como son los antibióticos. Ellos representan uno de los medicamentos más usados en las UCIs y que requieren relaciones $\mathrm{PK} / \mathrm{PD}$ definidas para emplearse.

Lo esperable es que la farmacocinética clínica se convierta en una herramienta con mayor utilización en el apoyo individualizado de las terapias en la UCI. Ello permitirá mejorar el uso de medicamentos y, a la vez, maximizar su eficacia y/o reducir las potenciales toxicidades. Se han elaborado y publicado variados estudios, pero restan aún muchos aspectos farmacocinéticos inciertos por aclarar.

\section{Referencias}

1. Introduction to clinical pharmacokinetics y basic pharmacokinetic principles. En: DiPiro J, Blouin R, Pruemer J, Spruill W. Concepts in clinical pharmacokinetics. Estados Unidos: Segunda edición. American Society of Health-System Pharmacists; 1996. p.1-40.

2. Boucher BA, Wood GC, Swanson JM. Pharmacokinetic changes in critically illness. Crit Care Clin 2006; 22 (2): 255-71.

3. Power BM, Forber AM, van Heerden PV, Ilett KF. Pharmacokinetics of drugs used in critically ill adults. Clin Pharmacokinet 1998; 34 (1): 25-56.

4. Varghese JM, Roberts JA, Lipman J. Pharmacokinetics and pharmacodynamics in critically ill patients. Curr Opin Anaesthesiol 2010; 23: 472-8.

5. Robinson S, Zincuk A, Strøm T, Larsen TB, Rasmussen B, Toft P. Enoxaparin, effective dosage for intensive care patients: double-blinded, randomized clinical trial. Crit Care 2010; 14 (2): R41.

6. Varghese JM, Roberts JA, Lipman J. Antimicrobial pharmacokinetic and pharmacodynamic issues in the critically ill with severe sepsis and septic shock. Crit Care Clin 2011; 27 (1): 19-34.

7. Pea F, Viale P, Furlanut M. Antimicrobial therapy in critically ill patients. A review of pathophysiological conditions responsible for altered disposition and pharmacokinetic variability. Clin Pharmacokinet 2005; 44 (10): 1009-34.

8. Liu P, Müller M, Derendorf H. Rational dosing of antibiotics: the use of plasma concentration versus tissue concentration. Int J Antimicrob Agents 2002; 19 (4): 285-90.

9. Lagneau F, Perbet S, Delefosse D, Wernet A, Stocco J, Marty J. Drugs pharmacokinetics in ICU patients: consequences of hypoalbuminemia upon drugs monitoring and dosing scheme. Intensive Care Med 2004; 30 (6): 1247. 
10. Park G. Molecular mechanisms of drug metabolism in the critically ill. Br J Anaesth 1996; 77 (1): 32-49.

11. Kirwan CJ, Lee T, Holt DW, Grounds RM, MacPhee IA, Philips BJ. Using midazolam to monitor changes in hepatic drug metabolism in critically ill patients. Intensive Care Med 2009; 35 (7): 1271-5.

12. Verbeeck RK, Musuamba FT. Pharmacokinetics and dosage adjustment in patients with renal dysfunction. Eur J Clin Pharmacol 2009; 65 (8): 757-73.

13. Greenberg E, Saad N, Abraham T, Balmir E. Drug dosage adjustment using renal estimation equations: a review of the literature. Hosp Pharm 2009; 44 (7): 577-84.

14. Regueira T, Andresen M, Mercado M, Downey P. Fisiopatología de la insuficiencia renal aguda durante la sepsis. Med Intensiva 2011; 35 (7): 424-32.

15. Udy AA, Roberts JA, Boots RJ, Peterson DL, Lipman J. Augmented renal clearance. Implications for antibacterial dosing in the critically ill. Clin Pharmacokinet 2010; 49 (1): 1-16.

16. Pea F, Viale P, Pavan F, Furlanut M. Pharmacokinetic considerations for antimicrobial therapy in patients receiving renal replacement therapy. Clin Pharmacokinet 2007; 46 (12): 997-1038.

17. Romero CM, Downey P, Hernández G. Hemofiltración de alto volumen en shock séptico. Med Intensiva 2010; 34 (5): 345-52.

18. Cornejo R, Downey P, Castro R, Romero C, Regueira T, Vega J, et al. High-volume hemofiltration as salvage therapy in severe hyperdynamic septic shock. Intensive Care Med 2006; 32 (5): 713-22.

19. Da Costa D, Gabrielli L, Andresen M, Downy P, Castro P. Shock cardiogénico por infarto agudo del miocardio manejado con hemofiltración de alto volumen: Caso clínico. Rev Med Chile 2009; 137: 1357-62.

20. Bilgrami I, Roberts JA, Wallis SC, Thomas J, Davis J, Fowler S, et al. Meropenem dosing in critically ill patients with sepsis receiving high-volume continuous venovenous hemofiltration. Antimicrob Agents Chemother 2010; 54 (7): 2974-8.

21. Boereboom FT, Ververs FF, Blankestijn PJ, Savelkoul TJ, van Dijk A. Vancomicyn clearance during continuous venovenous haemofiltration in critically ill patients. Intensive Care Med 1999; 25 (10): 1100-4.

22. del Mar Fernández de Gatta García M, Revilla N, Calvo MN, Domínguez-Gil A, Sánchez A. Pharmacokinetic/ pharmacodynamic analysis of vancomycin in ICU patients. Intensive Care Med 2007; 33 (2): 279-85.

23. Uchino S, Cole L, Morimatsu H, Goldsmith D, Bellomo R. Clearance of vancomycin during high-volume hemofiltration: impact of pre-dilution. Intensive Care Med 2002; 28 (11): 1664-7.
24. Dougnac A, Mercado M, Cornejo R, Cariaga M, Hernández $\mathrm{G}$, Andresen $\mathrm{M}$, et al. Prevalencia de sepsis grave en las Unidad de Cuidados Intensivos. Primer estudio multicéntrico. Rev Med Chile 2007; 135: 620-30.

25. O'Brien J, Ali N, Aberegg S, Abraham E. Sepsis. Am J Med 2007; 120 (2): 1012-22.

26. Morrell MR, Micek ST, Kollef MH. The management of severe sepsis and septic shock. Infect Dis Clin North Am 2009; 23 (3): 485-501.

27. Owens RC, Shorr AF. Rational dosing of antimicrobial agents: pharmacokinetic and pharmacodynamic strategies. Am J Health Syst Pharm 2009; 66 (12 Suppl. 4): S23-S30.

28. Lodise T, Drusano GL. Pharmacokinetics and Pharmacodynamics: optimal antimicrobial therapy in the intensive care unit. Crit Care Clin 2011; 27: 1-18.

29. Taccone F, Hites M, Beumier M, Scolleta S, Jacobs F. Appropriate antibiotic dosage levels in the treatments of severe sepsis and septic shock. Curr Infect Dis Rep 2011; 13 (5): 406-15.

30. Taccone F, Laterre PF, Dugernier T, Spapen H, Delattre I, Wittebole X, et al. Insufficient -lactam concentration in the early phase of severe sepsis and septic shock. Crit Care 2010; 14 (4): R126.

31. Roberts JA, Roberts MS, Semark A, Udy AA, Kirkpatrick $\mathrm{CM}$, Paterson DL, et al. Antibiotic dosing in the "at risk" critically ill patients: linking pathophysiology with pharmacokitenics/pharmacodynamics in sepsis and trauma patients. BMC Anesthesiol 2011; 11: 3 .

32. Giannoni E, Moreillon P, Cotting J, Moessinger A, Billie J, Décosterd L, et al. Prospective determination of plasma imipenem concentration in critically ill children. Antimicrob Agents Chemother 2006; 50 (7): 2563-8.

33. Belzberg H, Zhu J, Cornwell EE, Murray JA, Sava J, Sa$\lim \mathrm{A}$, et al. Imipenem levels are not predictable in the critically ill patients. J Trauma 2004; 56 (1): 111-7.

34. Roos JF, Bulitta J, Lipman J, Kirkpatrick CM. Pharmacokinetic-pharmacodynamic rationale for cefepime dosing regimens in intensive care units. J Antimicrob Chemother 2006; 58 (5): 987-93.

35. Touw D, Neef C, Thomson A, Vinks A. Cost-effectiveness of therapeutic drug monitoring: an update. EJHP Science 2007; 13 (4): 83-91.

36. Álvarez-Lerma F, Olaechea P, Grau S, Marín M, Domínguez A, Martínez-Lanao J, et al. Recomendaciones para la monitorización de antibióticos en pacientes críticos ingresados en UCI. Enferm Infecc Microbiol Clin 2008; 26 (4): 230-9.

37. Walson PD. Therapeutic drug monitoring in special populations. Clin Chem 1998; 44 (2): 415-9.

38. Grau S, Álvarez-Lerma F, Domínguez-Gil A. Pharma- 
cokinetic/pharmacodynamic indices: are we ready to use them in daily practice? Expert Rev Anti-Infect Ther 2007; 5(6): 913-6.

39. Martin J, Norris R, Barras M, Roberts JA, Morris R, Doogue $\mathrm{M}$, et al. Therapeutic Monitoring of vancomycin in adult patients. A consensus review of the American Society of Health-System Pharmacist, the Infectious Diseases Society of America, and the Society of Infectious Diseases Pharmacist. Clin Biochem Rev 2010; 31 (1): 21-4.

40. Milne S, Kenny G. Target controlled infusions. Curr Anaesth Crit Care 1998; 9 (4): 174-9.

41. Guarracino F, Lapolla F, Cariello C, Danella A, Doroni L, Baldassarri R, et al. Target Controlled Infusion: TCI.
Minerva Anestesiol 2005; 71 (6): 335-7.

42. Young CC, Prielipp RC. Benzodiazepines in the Intensive Care Unit. Crit Care Clin 2001; 17 (4): 843-62.

43. Spina SP, Ensom MH. Clinical pharmacokinetic monitoring of midazolam in critically ill patients. Pharmacotherapy 2007; 27 (3): 389-98.

44. Fragen RJ. Pharmacokinetics and pharmacodynamics of midazolam given via continuous intravenous infusion in intensive care units. Clin Ther 1997; 19 (3): 405-19.

45. Lee LS, Kinzig-Schippers M, Nafziger AN, Ma L, Sörgel F, Jones RN, et al. Comparison of 30-min and 3-h infusion regimens for imipenem/cilastatin and for meropenem evaluated by Monte Carlo simulation. Diagn Microbiol Infec Dis 2010; 68 (3): 251-8. 